\title{
Discussion on Nurses’ Psychological Health Echelon Management Model
}

\author{
Huang Yan, Chen Xiaomei, Zeng Xiangfa* \\ Chengdu Medical College, Chengdu, Sichuan 610500, China \\ *1362806408@qq.com
}

Keywords: psychological health; hospital management; nurses; echelon management

\begin{abstract}
At present, the tension between nurses and patients has brought large-scale interpersonal pressure for nurses. Coupled with pressure from work itself, personal development and competition, life, nurses have some psychological problems that lead to job burnout, and the final result is the loss of nursing talents. This paper analyzed the causes of nurses' psychological problems and the problems of hospital management. Then, echelon psychological management model is put forward, including age, difficulty, supervision and prevention, self-hematopoiesis and group psychological intervention.
\end{abstract}

\section{Introduction}

At present, there are prominent contradictions between nurses and patients [1-4]. Clinical nurses work in a long-term social environment where patients do not trust them, and their interpersonal pressure is also more obvious. Coupled with the complication and heavy responsibilities at work, nurses' psychological health problems are more common. Many scholars' surveys using psychological health self-assessment scale [5-9] show: nurses are significantly higher than normal in somatization, coercion, anxiety, depression, terror, hostility, interpersonal relationships and other sensitive factors, indicating that the overall psychological nurse health problems are prominent and common. Under long-term psychological pressure, nurses' job burnout is also more common. Job burnout is due to personal long-term job stress caused by occupation tiredness, questions toward their careers, and even wanting to quit and diverted. Surveys show that job burnout rates of nurses are generally high [10-12], and occupational burnout rates are even higher in emergency departments and critical illness departments.

Nurses' psychological problems come from many aspects, and the solution is primarily in hospital management. Nurses' psychological health echelon management methods are put forward in this article to explore new ways to solve the psychological health problems of nurses from hospital management point of view.

\section{Current nurses’ psychological health related issues}

\subsection{The source of nurses' psychological problems}

First, the unhealthy relationship between nurses and patients led to increased work pressure. At present, the contradiction between nurses and patients is prominent. Nurses and patients do not trust each other and guard each other, and minor things can lead to disputes between nurses and patients. Nurses need to spend more time and energy on their successful completion of routine clinical nursing tasks. In addition, nurses and patients often suffer from interpersonal pressures and even threats from patients due to some bickering and disputes on trivial matters. This inevitably affects the psychological health of nurses and leads to job burnout. And when the nurses are tired of work, it is often difficult to treat patients with a normal attitude, which affects the efficiency of work and may eventually lead to increased job errors and negligence.

\subsection{The pressure of clinical nursing work}

Complex clinical care is of technical high difficulty and heavy responsibility. Nurses have three 
shifts down work system, so the nurse's biological clock is often disrupted, and this irregular work and rest will also implicate the family. Clinical work is of $30 \%$ treatments and $70 \%$ nursing, showing the importance of nursing work. Therefore, nurses tend to have more work pressure. Combined with the salary of nurses is often lower than medical and technical staff, nurses psychological health may be more prominent and their burnout is higher than medical staff.

In addition nurses also face professional and technical promotion examinations, training, clinical nursing and teaching, nursing research tasks and many other pressures. The long-term pressure cannot often effectively be released and transferred, which gradually aggravates the psychological problems of nurses.

\subsection{Inadequate or lack of hospital psychological health management}

In response to the current psychological health problems and job burnout of nurses, many scholars have also done sufficient investigation. There are lots of data show that nurses psychological health problems are worthy of attention. Some scholars also put forward some countermeasures. However, on the whole, hospital's managers have not attached great importance to the psychological health of nurses, and many hospitals still lack special work in this area. The reason is currently lack of scientific management techniques. How to scientifically manage psychological health problems of nurses still lacks a set of scientific and easy mode. Hospitals want to start to solve this problem, but they often do not know how to start.

In general, there are many theoretical studies and few applied researches. Although some scholars have proposed to introduce EAP (Employee Assistance Program) [13-16] in hospitals to solve the psychological health problems of nurses and there are a few hospitals that are carrying out the practice, however, because of the implementation of EAP requires more psychologically specialized personnel, related materials and equipment, venues, etc., therefore, it is also a huge investment. Coupled with domestic people are not used to receiving western psychological counseling and it is not a decent thing to have any psychological problems, face problems make nurses often taboo their psychological problems. Therefore, EAP has not been recognized and promoted in hospitals.

\subsection{Nurses are lack of self-coping skills}

Part of the nurse's psychological problems is naturally in their own right. Self-psychological adjustment ability, contradictory treatment experience, healthy lifestyles and so on have an important relationship with the maintenance of psychological health. However, nurses have deficiencies in nurse-patient communication skills, methods of resolving contradictions, and self-regulation of psychology. These require additional learning or training to supplement. When encounter nurse-patient disputes, nurses take evasive behavior and do not take the initiative to actively deal with them as usual and it is often difficult to learn from the lessons.

In addition, heavy workload and negative psychology of work often affect nurse's family life. The disorder of life as a result of three shifts often affects the lives of family members. Therefore, nurses' life quality is also affected, in turn; the state of life will also affect the work status of nurses.

\section{Nurses’ psychological health echelon management model}

\subsection{Age echelon management model}

Nurses of different ages have different stresses in their work and life and different psychological problems. Therefore, according to age echelon division, we can make more targeted psychological intervention and management.

First echelon: 20-year-olds, including nurses aged 20-29. This age group is mostly young nurses who have just joined the workforce. As work environment and business are not yet familiar and skilled, there are not fully formed life rules, so their stress is mainly adaptation problems like orientation setbacks, adverse psychological and behavioral problems. General $\mathrm{PhD}$ graduates often have a wealth of life and clinical nursing experience. Young nurses often have not got married, so 
personal emotions, marriage; economic problems are often troublesome sources.

Second echelon: 30-year-old nurses, including 30-39 years old. At this stage, most nurses have become parents, and because children are still young, it is more important to raise children. Problems such as childcare, school attendance and children education, etc., are also factors tying down them in their work. As a result, they cannot devote themselves fully to their work.

Third echelon is 40-year-old age range, including 40-year-old to retirement age group. This stage nurses are more abundant in work and life experience, so the means to deal with work pressure is also more abundant. Their psychological pressure caused by their work pressure tends to be lighter. In psychological intervention, we often receive satisfactory results without too much energy.

In accordance with age echelon, the focus is on young nurses and different age groups have different pressures so they should be treated separately. As young nurses work and life have not yet a stable shape, individuals need to face more stresses and they are lack of relevant experience to deal with the problems technically, so young nurses are the focus of psychological management crowd.

\subsection{Difficulty echelon management mode}

Starting from the assessment of the seriousness of psychological problems of nurses, some nurses may have prominent psychological problems, which are the key points of psychological management. Others are good at self-regulation and do not intervene so as to effectively reduce the burden on hospital management.

The first echelon: heavy and difficult crowd. The stresses of these nurses often come from sudden work, life events and personal development frustrations. For example, negligence or misunderstanding encountered nurse-patient disputes, personal confidence in work has been hit harder; nurses family misconduct, divorce, life blow can directly affect nurses' work efficiency and enthusiasm. In addition, there is often tremendous competitive pressure on personal promotion. Once the competition fails, it will also cause obvious psychological blows to them, resulting in corresponding negative emotions and negative behaviors. Psychological problems of such people are often prominent, which is the focus of hospital psychological intervention.

The second echelon: Although they did not suffer any major setbacks and blows, the development of personal undertakings is sluggish. For some people and things, they often endure and it is often hard to see on the surface, but the heart is rather complicated. They have negative psychological and ideological situation but seldom expose. Major setbacks and strikes can easily lead to prominent psychological and behavioral problems to these nurses, and therefore they are the group hospital managers should focus on.

Third Echelon: nurses have family harmony and career development. These nurses' psychological problems occur less than normal, and they have a certain degree of affordability when subject to a variety of events, and they are usually not the focus of nursing psychological management.

Grasp the key psychological problem nurse groups, and the key of hospital psychological intervention. Psychological intervention must be targeted can make us do more with less. Otherwise, targeting the wrong group may cause nurse sensitivity and resistance.

\subsection{Echelon mode of prevention and control system}

It is necessary to carry out psychological health management work of nurses and discovery or diagnosis is essential. How to accurately grasp the psychological status of nurses is just as there is a diagnostic process before the treatment of patients, but the psychological evaluation of a large number of nurses is often complicated and difficult. Therefore, it is very important to establish a multilevel nurses' psychological management system for monitoring and evaluating their psychological health. The system can provide managers with the most cutting-edge and true conditions so as to help them make scientific decisions.

The first echelon takes department as a unit, department heads and managers have the responsibility to observe the nurses' psychological and behavioral conditions in their daily work; if necessary, understand the difficulties and worries, and timely report observed situation to hospital 
management. Each department can choose nurses with good capability to participate in psychological observation and assessment training as psychological health observers who are responsible for the observation and reporting tasks in the department.

The second echelon is psychology supervision. This refers to that personnel with psychology professional knowledge and skills come in the department and quietly observe the psychological state of nurses and make assessment report. Give priority to emergency department, ICU and other departments to observe. Observation report must propose solutions to the hospital management for reference. This part of observation work can be outsourced to professional counseling clinics, and psychologists from professional institutions can come to the hospital on a regular basis to observe quietly and make an assessment report. If possible, we can invite these agencies to participate in quarterly observation.

The third echelon is the senior managers' observation of hospital management. Nurses' psychological health management team or a special office should be established for regular visits to the department, rounds, and understanding of patient's clinical evaluation in hospitals or nurses in some specific evaluation. The field view is a good way to better grasp the work of nurses.

\subsection{Self-hematopoietic hierarchy model}

Self-hematopoiesis is to cultivate and strengthen nurses' self-psychological adjustment and communication ability with patients, thus helping them to reduce job frustration and reduce the administrative burden on the hospital.

First, cultivate nurses' communication abilities and habits with patients. By holding a series of training courses or continuing education programs, advanced management and service concepts and technologies in companies are introduced to enable nurses to work on-the-job to improve their communication skills and practice in practical work. For a long time, they will form a good habit of nurse-patient communication. Nurse-patient relationship begins from good communication into a virtuous circle to reduce nurse's pressure of interpersonal relationships, which can effectively enhance their psychological health.

Second, we must strengthen nurse psychology common sense of on-the-job training. In addition to psychological common sense, the training content also includes self-decompression skills. The main purpose of the program is to help nurses to recognize some of their own emotional problems and to master the ways and skills of depressing people in society so as to enhance their psychological self-regulation ability.

Third, strengthen nurses' skills in dealing with emotions and marriages. Emotions and marital problems are often sources of stress in life. Since life pressure can directly affect working status of nurses, it is of great significance to provide scientific guidance in terms of feeling and marriage. For example, we can invite love and marriage experts to the hospital for nurses to give lectures, answer to problems, and improve nurses' self-ability to deal with life issues.

In the invitation of professionals for experts lecture, the process should be recorded and make electronic version on the hospital website, for nurses to freely download, thereby reducing the frequency of experts invited to save the hospital management costs.

\subsection{Group psychological intervention management techniques}

The most direct way of nurses' psychological management is psychological intervention. However, psychological intervention cannot be one-on-one intervention, and group psychological intervention [17-18] often has high efficiency and save management costs. There is an additional function of group psychological intervention. It can effectively enhance the emotional communication between nurses and enhance their friendship so as to promote mutual assistance and cooperation in work and thus reduce errors in work.

First is to take department as a unit. We can organize dinner party, group outdoor tourism and other ways to strengthen inter-depart psychological harmony between staff. Having a good colleague relationship can enhance individual's tolerance to work pressure and setbacks.

The second is to carry out hospital-wide cultural activities. At the same time, strengthen the necessary places and facilities construction. For example, hold prize garden activities on festivals, 
sports competitions in spring and autumn, outstanding essay photography works selection activities. These can enrich the hospital's cultural life and an important measure to enhance the cohesion of the hospital. And these activities have a traditional basis and are therefore much more nurtured and engaged by nurses.

The third is to invite professional psychology staff to do group therapy. For example, carry out interesting psychological games in classroom and answer problems of medical staff. Through group psychological intervention, further strengthen the harmony between nurses.

Fourth is to hold nurse-patient associations. Invite part of successful cured special patients and the families of hospitalized patients to get involved. In a good atmosphere, provide communication platform for doctors and patients to enhance the interaction between nurses and patients, strengthen nurse-patient communication, enhance exchanges and win patient's reputation. And then ease and resolve the tension between nurses and patients.

In short, nurses' psychological health problems can be divided into multiple parts according to the degree of difficulty and age group. Targeted psychological management can often achieve a multiplier effect. Therefore, hospitals at all levels should pay attention to nurses' psychological health management, effectively maintain the psychological health of nurses and avoid the loss of nursing talents.

\section{Acknowledgments}

This work is supported by scientific research project "Qualitative research on the present application of humanistic care ability of nursing staff in Chengdu City" of the Education Department of Sichuan in 2017, Number: 17SB0110.

\section{References}

[1] Liu Youining. The root of sharp contradiction between doctor and patient [J]. Journal of Tuberculosis and Respiratory Diseases .2012,35 (6): 401-403

[2] Zhang Wenjuan, Hao Yanhua, Wu Qunhong. China's doctor-patient relationship tension and countermeasures [J] Medicine and Society .2014,27 (4): 44-46

[3] Chen Qianwen, Zheng Hong'e. Review of the relationship between doctors and patients at home and abroad [J]. Medicine and Philosophy: Humanities and Social Sciences .2014,35 (3): 44-48

[4] Zhou Shenglai. On medical system from doctor and patient's contradiction [J] Chinese Hospital .2013, (15): 90

[5] Zhang Gongzhen, Jiang Guanggen. Analysis of doctor's psychological health problems [J]. Medicine and Philosophy: Humanities and Social Sciences .2008,29 (8): 55-57

[6] Yan Bin, Chen Yuan, Li Fang. Survey and analysis of psychological health of nurses in general hospitals [J]. Journal of Kunming Medical University .2013,34 (11): 152-154

[7] Wu Xiuping. A top three hospital's ward nurses’ work stress and psychological health survey [J]. Chinese Medical Guide. 2013,13 (36): 293-294

[8] Xi Yonghong. Nurses psychological health status and related factors and countermeasures [J]. China Health Industry.2014,11 (3): 71-73

[9] Luo Guangqiong, $\mathrm{Pu}$ Jianwen, Li Bin. Psychological health and general practitioners' psychological health survey [J]. Guangdong Medical Journal. 2010,47 (1): 108-109

[10] Xie Jialing, Lan Wenyu, Yang Bin. Survey of occupational burnout among medical staff [J]. Sichuan psychological Health. 2013, 26 (2): 106-108

[11] Lu Siqi, Yan Jin, Tang Yunxiang. The relationship between occupational burnout and coping style of medical staff [J]. Journal of Clinical Psychosomatic Diseases .2012,18 (4): 340-343 
[12] Zhang Yan, Song Shenchao, Yang Xing. Study on occupational burnout of 2079 medical staffs [J]. Journal of Guiyang Medical College. 2011,36 (5): 485-487,490.

[13] Wang Jun. Employee help program [J]. Chinese Pharmacy. 2013,12 (22): 20-28

[14] Chen Jinshu. Exploration and thinking about carrying out employee help program [J]. Journal of Petrochemical Industry and Management College of China. 2013, 15 (4): 25-27, 39

[15] Zhong Jingyun. Cuixin Qi. EAP applications in hospital management [J]. Journal of Jilin Medical College .2011,32 (5): 292-294

[16] Zhang Qingling, Liu Minghua. The necessity and feasibility of introducing EAP in hospital management [J]. Journal of Laser Medicine. 2011, 32 (2): 96

[17] Zheng Huifang, Cao Xinmei, Nie Leiyan. Group psychological intervention's impact on psychiatric nurses psychological situation after suffered violence in work place [J]. Nursing Research: Second Edition.2013,26 (5): 1427-1429

[18] Qin Ruiyun, Zhang Yongqi, Wang Haixia. Group psychological intervention application in nursing team training [J]. Qiqihar Medical College. 2013,29 (19) 2939-2941 